\title{
The Quantum Mechanics as Also a Case of the Ether Elasticity Theory
}

\author{
David Zareski ${ }^{1}$
}

${ }^{1}$ I.A.I, Israel Aerospace Industries Yehud, Israel

Correspondence: David Zareski, Shilo Str. 74, Roch HaAyin, Israel. E-mail: zareski@inter.net.il

Received: April 8, 2014 Accepted: June 23, 2014 Online Published: July 1, 2014

doi:10.5539/apr.v6n4p48 URL: http://dx.doi.org/10.5539/apr.v6n4p48

\begin{abstract}
The Schrödinger equation ensues from the following axiom: in Cartesian coordinates and in absence of gravitation, to the component $p_{\mu},(\mu=1,2,3,4)$, of the momentum tensor is associated the operator $-\mathrm{i} \hbar \partial_{\mu}$. We show here this equation is a particular case of the equation that governs, even in presence of gravitation, the oscillatory displacements $\xi$ of the points of the ether shown to be a specific elastic medium. That is to say that the Schrödinger equation of which the solutions are the scalar state functions $\Theta$ is a particular case of the equation of the vectorial waves $\xi$ propagated in the ether. As shown in previous publications, a mobile particle is a superposition $\hat{\xi}$ of these waves $\xi$ that form a globule moving like this particle; here we show, in particular that, in a bound state, it is the interferences of these waves $\xi$ that creates the so called "quantum states". The ether elasticity theory therefore do not only generalizes the quantum mechanics, but also gives the physical signification of the quantum phenomena.
\end{abstract}

Keywords: ether elasticity theory, quantum mechanics, physical signification of the quantum effects

\section{Introduction}

We use the following notations. The Greek indexes take the values $1,2,3,4$, they are associated to spatial-time quantities. The Latin indexes take the values $1,2,3$, they are associated to spatial quantities. The index 4 is associated to time quantities. We denote by $\mathrm{p}_{\mu}$ the covariant components of the momentum tensor, by $\mathrm{x}^{\mu}$ the contra-variant coordinates, by $\dot{\mathrm{x}}^{\mu}$ the time derivative of $\mathrm{x}^{\mu}$, i.e., $\dot{\mathrm{x}}^{\mu} \equiv \mathrm{dx}{ }^{\mu} / \mathrm{dt}$, and by $\partial_{\mu}$ the partial derivatives $\partial / \partial x^{\mu}$.

The Schrödinger equation is based on the axioms inspired from the Broglie plane wave, postulating that "in Cartesian coordinates and in absence of gravitation, to the component $\mathrm{p}_{\mu}$ of the momentum tensor is associated, the operator $-\mathrm{i} \hbar \partial_{\mu}$ applied on the wave function ". Therefore, even though it yields very important results, the Schrödinger's equation is arbitrary since it is founded on these arbitrary axioms. Zareski (2011) shown that the quantum mechanics may be extended to the case where there is present a gravitational field, by replacing the operator $-\mathrm{i} \hbar \partial_{\mu}$, by the covariant operator $-\mathrm{i} \hbar \mathrm{D}_{\mu}$, but even this last axiom is arbitrary.

Therefore one may think that since it reposes on axioms, the quantum mechanics theory may be generalized by a theory based on time, space, forces, movements and general relativity. In that context, we show here that the quantum mechanics is a particular case of the "ether elasticity theory (Zareski, 2001, 2012, 2013)", compatible with the general relativity. One reminds that Maxwell and Einstein presumed its existence, indeed Maxwell wrote in Art. 866 of Maxwell (1954):

"Hence all these theories lead to the conception of a medium in which the propagation takes place, and if we

admit this medium as an hypothesis, I think it ought to occupy a prominent place in our investigations.". and Einstein, in Einstein (1920):

"Recapitulating, we may say that according to the general theory of relativity space is endowed with physical qualities; in this sense, therefore, there exists an ether. According to the general theory of relativity space

without ether is unthinkable; for in such space there not only would be no propagation of light, but also no possibility of existence for standards of space and time (measuring-rods and clocks), nor therefore any

space-time intervals in the physical sense.".

As shown in Zareski $(2001,2012,2013)$, the "ether elasticity equation" that governs the displacements $\xi$ of the points of the ether ensues from the Navier-Stokes-Durand equation of elasticity, that takes also into account the 
densities of couples applied to the elastic medium. Theses densities of couples were introduced by E. Durand, Cf. pp. 229-239 of Durand (1953), they complete the Navier-Stokes equation of elasticity.

In this paper one consider the particular case where the ether elasticity equation is defined by Equations (30)-(32) of Zareski (2013) and where the field of forces that act the particle is static, i.e., where as we show, the displacements $\xi$ of the points of the ether are waves of constant frequency. As shown here below, in this case the ether elasticity theory generalizes the quantum mechanics since it yields in particular the Schrodinger equation, but not only the Schrodinger equation of which the solutions are scalar state functions $\Theta$, but yields an equation of which the solution are vectorial waves $\xi$ called "particle waves". This new equation which ensues from the Navier-Stokes-Durand equation is valid even in the presence of a gravitational field.

These waves $\xi$ are called "particle waves" because they are associated to the $\operatorname{Par}(\mathrm{m}$, e)s, (i.e., particles of mass $\mathrm{m}$ and electric charge e), as following: an adequate superposition $\hat{\xi}$ of waves $\xi$ forms a globule that moves like a $\operatorname{Par}(\mathrm{m}, \mathrm{e})$, therefore a $\operatorname{Par}(\mathrm{m}, \mathrm{e})$ can be considered as such a globule, in particular a $\operatorname{Par}(0,0)$ is a photon and the waves $\xi$ associated it are electromagnetic; reciprocally, as we show here below, an adequate sum of such globules $\hat{\xi}$ forms a wave $\xi$, Cf. (Zareski, 2013). Mathematically, these sums are Fourier transforms and their inverses.

In a bound state of a $\operatorname{Par}(\mathrm{m}, \mathrm{e})$ submitted to a Schwarzschild field and to a Coulomb one due to a $\operatorname{Par}\left(\mathrm{m}_{0}, \mathrm{q}_{0}\right)$ immobile at the origin, the waves $\xi$ that compose $\hat{\xi}$ interfere with themselves. In this interference, only remain certain waves $\xi$ that do not destruct themselves in this interference, but on the contrary, are amplified, i.e., resonate. This resonance happens when a close trajectory of $\operatorname{Par}(m, e)$ contains an integer number "n" of wave lengths $I_{w}$, and these resonant waves are the equivalents of the so called "quantum state" of the quantum mechanics.

\section{Recalls on the Equation that Governs the Elastic Ether and of Its Solution $\xi$}

One considers that the fields to which is submitted a $\operatorname{Par}(\mathrm{m}, \mathrm{e})$ are static. In this case, Cf. Sec. V (ibid), the particle waves $\xi$ associated to such a $\operatorname{Par}(\mathrm{m}, \mathrm{e})$ is the solution of the following "ether elasticity wave equation"

$$
\operatorname{curl}\left(\mathrm{V}_{\mathrm{P}}^{2} \operatorname{curl} \xi\right)=\omega^{2} \xi
$$

where $V_{P}$ denotes the phase velocity of $\xi$, and $\omega$, a constant pulsation. As shown, (ibid), a solution $\xi$ of Equation (1) is of the form

$$
\xi=\xi_{0} \exp (i \phi) .
$$

where the phase $\phi$ is related to the Lagrange-Einstein function $L_{G}$ of a $\operatorname{Par}(m, m e)$ submitted to a gravitational and or a electromagnetic field by the relation

$$
\mathrm{d} \phi \equiv \mathrm{L}_{\mathrm{G}} \mathrm{dt} / \hbar,
$$

and where, Cf. Sec. II, (ibid),

$$
\mathrm{L}_{\mathrm{G}}=-\mathrm{mc \dot {s }}+\mathrm{eA}_{\mu} \dot{\mathrm{x}}^{\mu} / \mathrm{c} .
$$

In this expression, $A_{\mu}$ denotes the electromagnetic potential tensor and $\dot{\mathrm{s}} \equiv \mathrm{ds} / \mathrm{dt}$, where ds denotes the Einstein infinitesimal element. Furthermore, in (2), $\xi_{0}$ denotes a vector depending upon only the spatial coordinates $\mathrm{x}^{\mathrm{j}}$ of $\operatorname{Par}(\mathrm{m}, \mathrm{e})$. We give now the explicit expression for phase the $\phi$. Since the expression for the momentum tensor $\mathrm{p}_{\mu}$, defined by $\mathrm{p}_{\mu} \equiv \partial \mathrm{L}_{\mathrm{G}} / \partial \dot{\mathrm{x}}^{\mu}$, is,

$$
\mathrm{p}_{\mu}=-\mathrm{mcdx}_{\mu} / \mathrm{ds}+\mathrm{eA}_{\mu} / \mathrm{c},
$$

it follows that

$$
L_{G} d t=p_{\mu} d x^{\mu} \equiv p_{4} d x^{4}+p_{j} d x^{j} \equiv\left(-E_{T} d t+p_{j} d x^{j}\right) / \hbar .
$$

Now $V_{P}$ is defined by the fact that $\phi=0$, i.e., by

$$
V_{P}=d \ell / p_{j} d^{j},
$$

where $\mathrm{d} \ell$ denotes the infinitesimal length of its trajectory of the $\operatorname{Par}(\mathrm{m}, \mathrm{e})$. It follows that for a $\operatorname{Par}(\mathrm{m}, \mathrm{me})$ submitted to static fields, one has,

$$
\phi \equiv \omega\left(-\mathrm{t}+\int \mathrm{d} \ell / \mathrm{V}_{\mathrm{P}}\right),
$$

where the spatial curvilinear integral is taken along a trajectory defined in (Zareski, 2013), and where $V_{P}$, for which the expression is given here below for the cases that interest us, is the phase velocity of the wave $\xi$, and $\omega$ denotes the pulsation defined by 


$$
\omega \equiv \mathrm{E}_{\mathrm{T}} / \hbar .
$$

In Equation (4), $E_{T}$ denotes the total energy of the $\operatorname{Par}(m, e)$, this energy is constant since the fields to which it is submitted are static, furthermore $\mathrm{E}_{\mathrm{T}}$ can be written in the form

$$
\mathrm{E}_{\mathrm{T}} \equiv \mathrm{mc}^{2}+\mathrm{h} v \text {, }
$$

where $v$ is a constant frequency. As shown (ibid), for a $\operatorname{Par}(\mathrm{m}, \mathrm{e})$ such an electron or a proton, $\xi_{0}$ is perpendicular to the trajectory. Now the velocity $\mathrm{V}$ of $\operatorname{Par}(\mathrm{m}, \mathrm{e})$ is related to $\mathrm{V}_{\mathrm{P}}$ by the relation

$$
\frac{\partial}{\partial E_{T}}\left(\frac{E_{T}}{V_{P}}\right)=\frac{1}{V} .
$$

Therefore, if one defines $\phi^{\prime}$ by

$$
\phi^{\prime} \equiv \hbar \partial \phi / \partial \mathrm{E}_{\mathrm{T}},
$$

then, on account of Equation (3) and of (5a), one has

$$
\phi^{\prime}=-\mathrm{t}+\int \mathrm{d} \ell / \mathrm{V} .
$$

\section{The Schrodinger Equation as a Particular Form of the Ether Elasticity Equation}

When a $\operatorname{Par}(\mathrm{m}, \mathrm{e})$ is submitted to a Schwarzschild field and to a Coulomb field created by the $\operatorname{Par}\left(\mathrm{m}_{0}, \mathrm{q}_{0}\right)$, i.e., a particle of mass $m_{0}$ and electric charge $q_{0}$, immobile at the origin $\mathbf{O}$, the expression for $V_{P}$, is, (ibid),

$$
\mathrm{V}_{\mathrm{P}}^{2}=\frac{\mathrm{c}^{2} \mathrm{E}_{\mathrm{T}}^{2} \gamma^{2}}{\left.\gamma_{\mathrm{a}}^{2}\left[\left(\mathrm{E}_{\mathrm{T}}+\mathrm{eA}\right)_{4}\right)^{2}-\gamma^{2}\left(\mathrm{mc}^{2}\right)^{2}\right]},
$$

where,

$$
\gamma^{2} \equiv 1-\alpha / \mathrm{r}, \quad \alpha \equiv 2 \mathrm{~m}_{0} \mathrm{k} / \mathrm{c}^{2}, \quad \gamma_{\mathrm{a}}{ }^{2} \equiv 1+\alpha\left(\cos ^{2} \mathrm{a}\right) /\left(\mathrm{r}^{2}\right), \quad \text { eA } 4 \equiv-\left|\mathrm{eq}_{0}\right| /\left(4 \pi \varepsilon_{0} \mathrm{r}\right),
$$

"a" being the angle made by the radius vector $\mathbf{r}$ and the trajectory element of the $\operatorname{Par}(\mathrm{m}, \mathrm{e})$. From the identity

$$
\left(\mathrm{E}_{\mathrm{T}}+\mathrm{eA}_{4}\right)^{2}-\gamma^{2}\left(\mathrm{mc}^{2}\right)^{2} \equiv\left(2 \mathrm{mc}^{2}+\mathrm{hv}+\mathrm{eA}_{4}\right)\left(\mathrm{h} v+\mathrm{eA}_{4}\right)+\left(\mathrm{mc}^{2}\right)^{2} \alpha / \mathrm{r},
$$

it appears that, in the "non-relativistic approximation", i.e., when $\alpha / \mathrm{r}$ is neglected in front of 1 , and $\mathrm{h} v+\mathrm{eA}_{4}$ in front of $\mathrm{mc}^{2}$, but where $\left(\mathrm{mc}^{2}\right)^{2} \alpha / \mathrm{r}$ is not neglected, Equation (8) becomes

$$
\left(\mathrm{E}_{\mathrm{T}}+\mathrm{eA}\right)^{2}-\gamma^{2}\left(\mathrm{mc}^{2}\right)^{2} \cong 2 \mathrm{mc}^{2}[\mathrm{~h} v+\hat{\mathrm{a}} / \mathrm{r}],
$$

where $\hat{a}$ is defined by

$$
\hat{\mathrm{a}} \equiv\left|\mathrm{eq}_{0}\right| /(4 \pi \varepsilon)+\mathrm{mm}_{0} \mathrm{k},
$$

and the expression for the phase velocity (6) becomes then

$$
\mathrm{V}_{\mathrm{P}}^{2} \cong \frac{\mathrm{E}_{\mathrm{T}}^{2}}{2 \mathrm{~m}(\mathrm{~h} v+\hat{\mathrm{a}} / \mathrm{r})} .
$$

Now, denoting by $\boldsymbol{\psi}_{0}$ the vector defined by

Equation (2) can be written

$$
\boldsymbol{\psi}_{0} \equiv \xi_{0} \exp \left(\mathrm{i} \omega \int \mathrm{d} \ell / \mathrm{V}_{\mathrm{P}}\right)
$$

$$
\xi=\psi_{0} \exp (-i \omega t)
$$

inserting (13) in (1), one obtains

$$
\operatorname{curl}\left(\mathrm{V}_{\mathrm{P}}^{2} \operatorname{curl} \psi_{0}\right)=\omega^{2} \boldsymbol{\psi}_{0}
$$

By using the following two identities

$$
\operatorname{curl}(b \mathbf{B}) \equiv \operatorname{bcurlB}+\operatorname{gradb} \wedge \mathbf{B}, \quad \text { and } \operatorname{curl}(\operatorname{curl} B) \equiv-\nabla^{2} \mathbf{B}+\operatorname{grad}(\operatorname{div} \mathbf{B}),
$$

where "b" is any scalar and $\mathbf{B}$, any vector, Equation (14) takes the form 


$$
-\nabla^{2} \boldsymbol{\psi}_{0}+\boldsymbol{\Xi}=\frac{\omega^{2}}{\mathrm{~V}_{\mathrm{P}}^{2}} \boldsymbol{\psi}_{0}
$$

where, $\boldsymbol{\Xi}$ is defined by

$$
\boldsymbol{\Xi} \equiv\left[\operatorname{grad} .\left(\log \mathrm{V}_{\mathrm{P}}^{2}\right)\right] \wedge \operatorname{curl} \boldsymbol{\psi}_{0} .
$$

For the $\operatorname{Par}(\mathrm{m}, \mathrm{e})$ submitted the field created by this $\operatorname{Par}\left(\mathrm{m}_{0}, \mathrm{q}_{0}\right)$, then, on account of (6), Equation (16) becomes

$$
-\nabla^{2} \boldsymbol{\psi}_{0}+\boldsymbol{\Xi}=\left(\frac{\gamma_{a}}{c \gamma \hbar}\right)^{2}\left[\left(E_{T}+e A_{4}\right)^{2}-\gamma^{2}\left(m c^{2}\right)^{2}\right] \boldsymbol{\psi}_{0},
$$

where, in $\boldsymbol{\Xi}$ and in $\boldsymbol{\psi}_{0}, \mathrm{~V}_{\mathrm{P}}$ is given by (6). Yet, in the non-relativistic approximation, then, on account of (11), this Equation (18) becomes,

$$
-\nabla^{2} \boldsymbol{\psi}_{0}+\boldsymbol{\Xi} \cong \frac{2 \mathrm{~m}}{\hbar^{2}}(\mathrm{~h} v+\hat{\mathrm{a}} / \mathrm{r}) \boldsymbol{\psi}_{0},
$$

where now, in the expressions for $\boldsymbol{\Xi}$ and $\boldsymbol{\psi}_{0}, \mathrm{~V}_{\mathrm{P}}$ is given by (11), that is to say that if we denote

$$
\Phi \equiv \frac{\sqrt{2 \mathrm{~m}}}{\hbar} \int(\mathrm{h} v+\hat{\mathrm{a}} / \mathrm{r}) \mathrm{d} \ell,
$$

then (17) becomes

$$
\boldsymbol{\Xi}=-[\operatorname{grad} \log (\mathrm{h} v+\hat{\mathrm{a}} / \mathrm{r})] \wedge \operatorname{curl}\left[\xi_{0} \exp (\mathrm{i} \Phi)\right] .
$$

Let us now consider more specially this expression for $\mathbf{\Xi}$ given in (21). First, one has

$$
\operatorname{grad} \log (h v+\hat{a} / r)=-\frac{\hat{a}}{(h v+\hat{a} / r)} \frac{r}{r^{3}},
$$

furthermore, by using the left identity (15), and considering that

$$
\operatorname{grad} \exp (\mathrm{i} \Phi)=\left(\mathrm{i} \omega \mathbf{u} / \mathrm{V}_{\mathrm{P}}\right) \exp (\mathrm{i} \Phi)
$$

where $\mathbf{u}$ is the unitary vector along the trajectory of the $\operatorname{Par}(\mathrm{m}, \mathrm{e})$ at its position, one has

$$
\operatorname{curl}\left[\xi_{0} \exp (i \Phi)\right] \equiv \exp (i \Phi)\left[\operatorname{curl} \xi_{\mathbf{0}}+i \frac{\sqrt{2 m}}{\hbar}(h v+\hat{a} / r) \mathbf{u} \wedge \xi_{\mathbf{0}}\right] .
$$

Yet, since the only condition that $\xi_{0}$ has to satisfy is to be perpendicular to the plane of the trajectory of $\operatorname{Par}(\mathrm{m}, \mathrm{e})$, one can take $\xi_{0}$ to be also constant, in this case (21) becomes

$$
\boldsymbol{\Xi}=\mathrm{i} \frac{\sqrt{2 \mathrm{~m}}}{\hbar} \frac{\hat{\mathrm{a}}}{\mathrm{r}^{3}} \exp (\mathrm{i} \Phi) \mathbf{r} \wedge\left(\mathbf{u} \wedge \xi_{\mathbf{0}}\right),
$$

and $\mathbf{r} \wedge\left(\mathbf{u} \wedge \xi_{0}\right)$ becomes $-(\mathbf{u} \cdot \mathbf{r}) \xi_{0}$, it follows finally that

$$
\boldsymbol{\Xi} \cong-\mathrm{i} \frac{\sqrt{2 \mathrm{~m}}}{\hbar} \frac{\hat{\mathrm{a}}}{\mathrm{r}^{3}}(\mathbf{u} \cdot \mathbf{r}) \boldsymbol{\Psi}_{0} .
$$

Now, one sees that

$$
|\Xi|<<\left|\frac{2 \mathrm{~m}}{\hbar^{2}}\left(\mathrm{~h} v+\frac{\hat{\mathrm{a}}}{\mathrm{r}}\right) \Psi_{0}\right|,
$$

and moreover, that in a circular bound state then $\mathbf{u}$ and $\mathbf{r}$ are orthogonal, i.e., $\mathbf{u} \cdot \mathbf{r}=0$, i.e., $\boldsymbol{\Xi}=0$. It follows that (19) can be written with a good approximation, considering that $\hat{a} / \mathrm{r} \cong \mathrm{eA}_{4}$, 


$$
-\frac{\hbar^{2}}{2 \mathrm{~m}} \nabla^{2} \psi_{0} \cong \mathrm{eA}_{4} \psi_{0}+\mathrm{h} v \psi_{0},
$$

Now, multiplying the tow members of (28) by $\exp (-i 2 \pi v t)$ and denoting

$$
\psi \equiv \Psi_{0} \exp (-\mathrm{i} 2 \pi v \mathrm{t})
$$

Equation (28) becomes

that can be written as following

$$
-\frac{\hbar^{2}}{2 \mathrm{~m}} \nabla^{2} \psi \cong \mathrm{eA}_{4} \psi+\mathrm{h} v \psi
$$

$$
-\frac{\hbar^{2}}{2 \mathrm{~m}} \nabla^{2} \psi \cong \mathrm{eA}_{4} \psi+\mathrm{i} \hbar \partial_{\mathrm{t}} \psi
$$

Equation (28a) is Schrodinger's equation. We have therefore demonstrated that this equation is a particular form of the ether elasticity equation (1), i.e., that: the ether elasticity theory generalizes the quantum mechanics.

\section{Some Other Known Results Ensuing Also From the Ether Elasticity Theory}

\subsection{The Bohr-Sommerfeld Condition}

For simplicity we consider a $\operatorname{Par}(\mathrm{m}, \mathrm{e})$ submitted only to a electrostatic field created by a $\operatorname{Par}\left(\mathrm{m}_{0}, \mathrm{q}_{0}\right)$ and use as in Zareski (2013), the following notations: if $\omega$ is fixed, and $\xi, \phi$, and $\phi^{\prime}$ are functions of $\omega+\Delta \omega$, then they will be denoted $\xi(\Delta \omega), \phi(\Delta \omega)$, and $\phi^{\prime}(\Delta \omega)$, and if $\Delta \omega=0$, then they will be denoted simply $\xi, \phi$, and $\phi^{\prime}$. Following the finite increment theorem one has

$$
\phi(\Delta \omega)=\phi+\Delta \omega \phi^{\prime}+\frac{1}{2}(\Delta \omega)^{2} \phi^{\prime \prime}(\theta \Delta \omega),
$$

where $\phi^{\prime \prime}(\theta \Delta \omega)$ is the value of $\partial \phi^{\prime} / \partial \omega$, when $\omega$ takes the value $\omega+\theta \Delta \omega,(0<\theta<1)$. Now like in Zareski (2013), we show now that $(\Delta \omega)^{2} \phi^{\prime \prime}(\theta \Delta \omega)$ is very small in front of $\Delta \omega \phi^{\prime}$, i.e., is negligible, indeed one can verify that in absence of gravitation, then

$$
\phi^{\prime \prime}(\theta \Delta \omega)=-\frac{\hbar}{\mathrm{c}}\left(\mathrm{mc}^{2}\right)^{2} \int \frac{\mathrm{d} \ell}{\left[\left(\mathrm{E}_{\mathrm{T}}+\mathrm{eA}_{4}\right)^{2}-\left(\mathrm{mc}^{2}\right)^{2}\right]^{3 / 2}} .
$$
Considering that $\mathrm{m}$ is the mass of the electron, one has, in MKSA unit, $\frac{\hbar}{\mathrm{c}}\left(\mathrm{mc}^{2}\right)^{2} \cong 10^{-56}$, therefore one can
write with a very good approximation, as in Zareski (2013) that

$$
\phi(\Delta \omega)=\phi+\Delta \omega \phi^{\prime},
$$

i.e.,

$$
\xi(\Delta \omega)=\xi \exp \left(\mathrm{i} \Delta \omega \phi^{\prime}\right)
$$

and that a $\operatorname{Par}(\mathrm{m}, \mathrm{e})$ is a superposition $\hat{\xi}(\Delta \omega)$ of waves $\xi$ defined by

for which the explicit expression is,

$$
\hat{\xi}(\Delta \omega) \equiv \frac{1}{\Delta \omega} \int_{-\Delta \omega / 2}^{\Delta \omega / 2} \xi(\vartheta) \mathrm{d} \vartheta
$$

$$
\hat{\xi}(\Delta \omega)=\xi_{0} \exp \left[i \omega\left(-\mathrm{t}+\int \frac{\mathrm{d} \ell}{\mathrm{V}_{\mathrm{P}}}\right)\right] \operatorname{SINC}\left[\frac{\Delta \omega}{2}\left(-\mathrm{t}+\int \frac{\mathrm{d} \ell}{\mathrm{V}}\right)\right],
$$

where, since the maximum of $\operatorname{SINC}\left[\frac{\Delta \omega}{2}\left(-t+\int \frac{\mathrm{d} \ell}{\mathrm{V}_{0}}\right)\right]$ which is of small volume moves with the velocity $\mathrm{V}$. 
In a bound state of a $\operatorname{Par}(\mathrm{m}, \mathrm{e})$ submitted to a Schwarzschild field and to a Coulomb one, due to $\operatorname{Par}\left(\mathrm{m}_{0}, \mathrm{q}_{0}\right)$, the waves $\xi$ that compose $\hat{\xi}$ interfere with themselves. In this interference, only reminds a certain wave $\xi$ that do not destruct itself in this interference, but on the contrary, resonates. This resonance happens when a close trajectory of $\operatorname{Par}(m, e)$ contains an integer number " $n$ " of wave lengths $I_{w}$.

Let us consider the case where the trajectories described by $\operatorname{Par}(\mathrm{m}, \mathrm{e})$ attracted by $\operatorname{Par}\left(\mathrm{m}_{0}, \mathrm{q}_{0}\right)$ are circular, and located in the plane $\theta=\pi / 2$. In such a circular state, $I_{w}$ is constant since it do not depend upon the angle $\varphi$ described by the constant radius vector $r$ denoted then $\rho$. The resonance condition is then such that

$$
2 \pi \rho / I_{w}=n .
$$

Now the expression for the total frequency $v_{T}$ is

$$
v_{\mathrm{T}}=\mathrm{E}_{\mathrm{T}} / \mathrm{h},
$$

and since $I_{w}=V_{P} / v_{T}$, the expression for $I_{w}$ is

$$
\mathrm{I}_{\mathrm{w}}=\mathrm{hV}_{\mathrm{P}} / \mathrm{E}_{\mathrm{T}} \text {. }
$$

In this circular case, we can express $V_{P}$ as a function of the component $p_{\varphi}$ of the momentum tensor defined by

$$
\mathrm{p}_{\varphi} \equiv \partial \mathrm{L}_{\mathrm{Gc}} / \partial \dot{\varphi},
$$

where $\mathrm{L}_{\mathrm{Gc}}$ is the Lagrange-Einstein function in this case. Indeed, in this case where the trajectories are circumferences of radius $\rho$ in the plane $\theta=\pi / 2$, the expression for $\mathrm{L}_{\mathrm{Gc}}$ is

$$
\mathrm{L}_{\mathrm{Gc}}=-\mathrm{mcs}_{\mathrm{c}}+\mathrm{eA} \dot{\mathrm{x}}^{4} / \mathrm{c} \text {, }
$$

where

$$
\dot{\mathrm{s}}_{\mathrm{c}}=\sqrt{\gamma^{2}\left(\dot{\mathrm{x}}^{4}\right)^{2}-\dot{\varphi}^{2} \rho^{2}},
$$

From (2) and (3), one sees that $V_{p}=E_{T} / p_{j} u^{j}$, and since $\rho$ is constant, it follows that

$$
p_{j} u^{j}=p_{\varphi} d \varphi /(\rho d \varphi) \equiv p_{\varphi} / \rho
$$

therefore, $V_{P}$ can be written in the following form

$$
\mathrm{V}_{\mathrm{P}}=\mathrm{E}_{\mathrm{T}} \rho / \mathrm{p}_{\varphi}
$$

and $I_{w}$ as following

$$
\mathrm{I}_{\mathrm{w}}=\mathrm{h} \rho / \mathrm{p}_{\varphi} .
$$

Inserting this expression for $I_{w}$ in (29), one retrieves the Bohr-Sommerfeld relation,

$$
\mathrm{p}_{\varphi}=\mathrm{n} \hbar,
$$

which is a resonance condition for the wave $\xi$ defined in (2) or (3), in that circular bound state.

4.2 The Permitted, (Eigen), Values of $r$ and hv

We determine now the values of $v$ and $\rho$ that cause this resonance by determining two equations involving them. From (32)-(34), it follows that, in this circular bound state,

$$
\mathrm{p}_{\varphi}=\mathrm{mc} \dot{\varphi} \rho^{2} / \dot{\mathrm{s}}_{\mathrm{c}} \equiv \mathrm{mc} \rho \mathrm{V} / \dot{\mathrm{s}}_{\mathrm{c}} \text {. }
$$

Now, in this space-time, the expression for the velocity $\mathrm{V}$ in this circular motion, is, (ibid),

$$
\mathrm{V}=\frac{\mathrm{c} \gamma}{\left(\mathrm{mc}^{2}+\mathrm{h} v+\mathrm{eA}_{4}\right)} \sqrt{\left(\mathrm{mc}^{2}+\mathrm{h} v+\mathrm{eA}_{4}\right)^{2}-\gamma^{2}\left(\mathrm{mc}^{2}\right)^{2^{2}}},
$$

which, in the non-relativistic approximation, becomes, considering (9),

$$
V \cong \sqrt{\frac{2}{m}\left(h v+\frac{\hat{a}}{\rho}\right)} .
$$

It follows, by inserting (40), in (38), that

$$
p_{\varphi} \cong \rho \sqrt{2 m(h v+\hat{a} / \rho)},
$$

i.e., considering (37), one obtains 


$$
(\mathrm{n} \hbar)^{2} \cong 2 \mathrm{~m} \rho^{2}(\mathrm{~h} v+\hat{\mathrm{a}} / \rho) .
$$

Equation (42) connects $v$ and $\rho$, and, on account of (40), yields

$$
\mathrm{V}^{2} \cong\left(\frac{\mathrm{n} \hbar}{\rho \mathrm{m}}\right)^{2}
$$

In order to determine another equation of $v$ and or of $\rho$, we use the following other motion equation

$$
\frac{\mathrm{d}}{\mathrm{dt}} \frac{\partial \mathrm{L}_{\mathrm{Gc}}}{\partial \dot{\mathrm{r}}}-\frac{\partial \mathrm{L}_{\mathrm{Gc}}}{\partial \mathrm{r}}=0 \text {. }
$$

From (33), one sees that $\partial \mathrm{L}_{\mathrm{Gc}} / \partial \dot{\mathrm{r}}=0$, it follows also that $\partial \mathrm{L}_{\mathrm{Gc}} / \partial \mathrm{r}=0$, therefore (44) becomes, considering (33),

$$
-\frac{1}{2} \mathrm{mc} \frac{\alpha \mathrm{c}^{2} / \rho^{2}-2 \dot{\varphi}^{2} \rho}{\sqrt{\gamma^{2} \mathrm{c}^{2}-\mathrm{V}^{2}}}+\mathrm{e} \partial_{\rho} \mathrm{A}_{4}=0 .
$$

Keeping in mind that $\dot{\varphi} \rho=\mathrm{V}$, and that $\partial \mathrm{A}_{4} / \partial \rho=\mathrm{q}_{0} /\left(4 \pi \varepsilon_{0} \rho^{2}\right)$, (45) becomes, in the non-relativistic approximation,

$$
m \rho V^{2}-\hat{a} \cong 0,
$$

and taking into account (43) and (10), one obtains

$$
\rho \cong(\mathrm{n} \hbar)^{2} /(\hat{a} m) .
$$

Inserting (47) into (42), one obtains

$$
\mathrm{h} v=-\frac{1}{2} \frac{\hat{\mathrm{a}}^{2} \mathrm{~m}}{(\mathrm{n} \hbar)^{2}},
$$

and since $\left|\mathrm{eq}_{0}\right| /(4 \pi \varepsilon)>>\mathrm{mm}_{0} \mathrm{k}$, Equation (48) can be written also with a good approximation

$$
\mathrm{h} v=-\frac{1}{2} \frac{\mathrm{m}}{(\mathrm{n} \hbar)^{2}}\left(\frac{\mathrm{eq}_{0}}{4 \pi \varepsilon}\right)^{2} .
$$

One sees that the relation (49) and (47), obtained from the theory of the waves $\xi$ in the elastic ether are the same as those obtained from the current quantum mechanics theory. It follows again that there is a relation between these two theories and, moreover, that the ether elasticity theory generalizes the usual quantum mechanics theory.

\section{Conclusions}

Therefore the ether elasticity theory here presented only in the case of the Schrodinger and of circular quantum states can yield more results than only the Schrodinger equation since it lies on the physical properties of a specific elastic medium: the ether in which propagate waves and where the fields are changes in the elastic properties of this medium. Finally both the ether elasticity theory and the quantum mechanics theory yield the Schrodinger equation, but this equation is only a particular result of the ether elasticity theory.

In a next paper we will show that the electron spin ensues also from this ether elasticity theory.

\section{References}

Durand, E. (1953). Electrostatique et Magnétostatique. (Masson \& Cie, 1953).

Einstein, A. (1920). Ether and the theory of relativity: The collected papers of Albert Einstein. Berlin: Springer.

Maxwell, J. C. (1954). A Treatise on Electricity and Magnetism (Dover, New-York, 1954).

Zareski, D. (2001). The elastic interpretation of electrodynamics. Found. Phys. Lett., 14, 447.

Zareski, D. (2011). Progress in Relativity, Gravitation, Cosmology. Nova Science Publisher, Inc.

Zareski, D. (2012). On the elasto-undulatory interpretation of fields and particles. Physics Essays, 25(2), 268-277. 
Zareski, D. (2013). Fields and wave-particle reciprocity as changes in an elastic medium: The ether. Physics Essays, 26(2), 288-295.

\section{Copyrights}

Copyright for this article is retained by the author(s), with first publication rights granted to the journal.

This is an open-access article distributed under the terms and conditions of the Creative Commons Attribution license (http://creativecommons.org/licenses/by/3.0/). 